\title{
Assimetria na relação escavação/disco óptico em adolescentes
}

\author{
Cup-to-disc ratio asymmetry in adolescents
}

\author{
Maria Elizabete Jimenes de Campos ${ }^{1}$ \\ Denny Marcos Garcia ${ }^{2}$ \\ Maria de Lourdes Veronese Rodrigues ${ }^{3}$
}

\section{RESUMO}

Objetivo: Verificar a frequência de assimetrias superiores a 0,2 na razão escavação/disco em uma população de adolescentes. Métodos: Retinografias de ambos os olhos de 123 jovens foram digitalizadas na resolução de 300 pixels por polegada (300 dpi), 8 bits, padrão de cor RGB. Para a realização das medidas foi utilizado o software Image J, versão 1.42, desenvolvido pelo National Institute of Health (NIH), plataforma Java versão 1.6. Resultados: Os valores das diferenças, entre os dois olhos do mesmo indivíduo, na razão das medidas do diâmetro vertical da escavação/ diâmetro vertical do disco óptico variaram de 0 a 0,30 . A média das diferenças foi 0,07 , a mediana 0,06 e o desvio padrão 0,05 . Assimetrias de até 0,06 apresentaram frequência de $54,4 \%$ e assimetrias de até 0,1 estiveram presentes em 79,7\% da amostra. Dois indivíduos (1,63\%) apresentaram assimetrias superiores a 0,2. Conclusão: A frequência de assimetrias no grupo de adolescentes estudado é similar à encontrada em estudos realizados com população adulta normal.

Descritores: Glaucoma/diagnóstico; Disco óptico/patologia; Doenças do nervo óptico; Fundo de olho; Campos visuais; Retina; Fotografia/métodos; Adolescentes

Departamento de Oftalmologia, Otorrinolaringologia e Cirurgia de Cabeça e Pescoço da Faculdade de Medicina de Ribeirão Preto da Universidade de São Paulo (USP) - Ribeirão Preto.

${ }^{1}$ Pós-graduanda, nível Mestrado, do Programa de PósGraduação em Oftalmologia, Otorrinolaringologia e Cirurgia de Cabeça e Pescoço da Faculdade de Medicina de Ribeirão Preto da Universidade de São Paulo (USP) - Ribeirão Preto (SP) - Brasil. Professora Assistente de Oftalmologia da Faculdade de Medicina de $\mathrm{Ca}-$ tanduva - Faculdades Integradas Padre Albino - Catanduva (SP) - Brasil.

2 Pós-graduando, nível Doutorado, do Programa de PósGraduação em Oftalmologia, Otorrinolaringologia e Cirurgia de Cabeça e Pescoço da Faculdade de Medicina da USP - Ribeirão Preto (SP) - Brasil.

${ }^{3}$ Professora Titular do Departamento de Oftalmologia, Otorrinolaringologia e Cirurgia de Cabeça e Pescoço da Faculdade de Medicina da USP - Ribeirão Preto (SP) - Brasil.

Endereço para correspondência: Maria de Lourdes Veronese Rodrigues. Departamento de Oftalmologia, Otorrinolaringologia e Cirurgia de Cabeça e Pescoço Faculdade de Medicina de Ribeirão Preto. Universidade de São Paulo. Hospital das Clínicas, $12^{\circ}$ andar Campus USP - Ribeirão Preto (SP) CEP 14049-900

E-mail: mdlvrodr@fmrp.usp.br

Recebido para publicação em 19.11.2009

Última versão recebida em 15.04.2010

Aprovação em 26.05.2010

\section{INTRODUÇÃOO}

Glaucoma é a maior causa de cegueira não curável no mundo ${ }^{(1)}$. Em 2003, o Conselho Brasileiro de Oftalmologia estimava que no Brasil houvessem 900 mil portadores dessa doença, e que, provavelmente, 720 mil estavam assintomáticos, ainda necessitando de diagnóstico ${ }^{(2)}$.

É consenso que no Brasil a forma predominante da doença é o glaucoma primário de ângulo aberto (GPAA) que, pelas suas características, não é passível de detecção antes que ocorram alterações da cabeça do nervo óptico e, ou da camada de fibras nervosas da retina. No entanto, por meio desses sinais, pode-se fazer a suspeita de glaucoma, antes que ocorram perdas do campo visual.

Por isso, é preconizado que os rastreamentos de glaucoma pré-perimétrico, em populações ou na prática clínica, iniciem com o exame da cabeça do nervo óptico ${ }^{(3-5)}$. Mesmo com a utilização de oftalmoscopia direta, em olhos com pigmentação normal, é possível verificar, também, lesões na camada de fibras nervosas, que podem preceder as do nervo óptico e geralmente aparecem vários anos antes das perdas no campo visual. Portanto, pode haver diferença entre a progressão estrutural (nervo óptico e camada de fibras nervosas) e a progressão funcional (campo visual) do glaucoma $^{(6-8)}$.

As escavações fisiológicas variam de um indivíduo para outro, dependendo da dimensão do canal da esclerótica. Na maioria dos indivíduos
\end{abstract}


existe simetria, entre os olhos, da relação escavação/disco, sendo assimetria importante sinal na suspeita de lesão glaucomatosa $^{(9-12)}$. Diferenças de razão escavação/disco de 0,2 podem aparecer em 1 a $6 \%$ da população adulta normal e assimetrias de 0,3 em $1 \%$ da população geral $^{(12-15)}$.

Apesar da diversidade de estudos realizados em adultos e dos relatos de alguns estudos que incluíram crianças e adolescentes ${ }^{(16-18)}$ a literatura, ainda, carece de dados referentes à medida escavação/disco em jovens na faixa etária de 14 a 18 anos de idade, época da vida em que ocorrem grandes mudanças no organismo, inclusive no globo ocular.

Assim, o objetivo do presente estudo foi verificar a frequência de assimetrias entre os olhos superiores a 0,2 na razão escavação/disco de uma população de adolescentes.

\section{MÉTODOS}

O projeto deste estudo, observacional e transversal, foi aprovado pelo "Comitê de Ética em Pesquisa" da Faculdade de Medicina de Catanduva - Faculdades Integradas Padre Albino, Catanduva, SP.

Apesar de frequentarem diferentes escolas públicas, todos os participantes pertenciam a uma mesma instituição, a "Legião Mirim de Catanduva" entidade, mantida pelo "Lions Clube de Catanduva", que tem por finalidade a inclusão social de menores.

Concordaram em colaborar com o trabalho 133 jovens com idades entre 15 anos e 18 anos, com desenvolvimento típico e pertencentes a diferentes etnias. A concordância em participar da pesquisa foi confirmada pela assinatura do Termo de Consentimento Livre e Esclarecido pelo responsável legal do menor.

Dentre os 123 adolescentes examinados, a maioria era do sexo feminino $(77=62,6 \%)$, com idade média $16,16(\mathrm{dp}=$ $\pm 0,54$ anos). Os participantes do sexo masculino tinham idade média semelhante (média \pm DP; 16,17 $\pm 0,61$ anos) correspondente a $37,4 \%$.

O critério de exclusão foi a presença de doenças oculares que impedissem a fixação ocular ou opacificações oculares.

Após midríase e cicloplegia, obtidas com a instilação de cloridrato de fenilefrina $10 \%$ solução oftálmica estéril (Allergan ${ }^{\circledR}$, Guarulhos-SP) e de tropicamida $1 \%$ (Mydriacyl ${ }^{\circledR}$, Alcon, São Paulo, Brasil), foram realizadas retinografias de ambos os olhos de todos os participantes, utilizando-se Retinógrafo Topcon $^{\circledR}$ (modelo 50 X, Tóquio, Japão), o qual utiliza o filme Pro Image 100 Kodak $^{\circledR}$ Profissional. As fotos foram reveladas no aparelho Minilab QSS 430 (Noritsu, Japão) e impressas na impressora Minilab Digital QSS $3001^{\circledR}$ (Noritsu, Japão).

Posteriormente, as retinografias foram digitalizadas na resolução de 300 pixels por polegada (300 dpi), 8 bits, padrão de cor RGB, através de "scanner" comercial Hewlett Packard modelo F4280 (Sorocaba, SP, Brasil). O "software” livre específico para processamento de imagens, Image J, versão 1.42, desenvolvido pelo National Institute of Health (NIH), plataforma Java versão 1.6 , foi utilizado para realização das medidas.
Cada imagem foi importada do banco de dados instalado no software a partir da digitalização das retinografias. Logo após, foi medido, em pixels, o diâmetro vertical da escavação do disco óptico e o diâmetro vertical do disco óptico. A partir daí, foi calculada a razão da medida do diâmetro vertical da escavação do disco óptico sobre a medida do diâmetro vertical do disco óptico, para cada um dos olhos de cada paciente (Figura 1). Assim, foi obtido um valor adimensional entre 0 e 1 para cada um dos olhos de cada paciente.

As medidas foram realizadas com a participação de, pelo menos, dois dos autores.

Foram, também, realizadas medidas da refração, no aparelho Auto-kerato-refractometer KR 7000, Topcon ${ }^{\circledR}$ (Paramus, NJ, USA), utilizando-se o equivalente esférico de cada olho.

\section{RESULTADOS}

Os valores das diferenças, entre os dois olhos do mesmo indivíduo, da razão das medidas do diâmetro vertical da escavação/diâmetro vertical do disco óptico variaram de 0 a 0,30 (mediana=0,06) (Gráfico 1).

Assimetrias de até 0,06 apresentaram frequência de 54,4\% e assimetrias de até 0,1 estiveram presentes em 79,7\% da amostra. Dois participantes $(1,63 \%)$ apresentam assimetrias superiores a 0,2 , sendo a assimetria de um deles $0,3(0,83 \%$ dos participantes).

Os diâmetros verticais dos discos ópticos do indivíduo que apresentou assimetria de 0,21 foram: $O D=212,01$ pixels e $\mathrm{OE}=242,49$; $\mathrm{E} / \mathrm{D}: \mathrm{OD}=0,19$ e $\mathrm{OE}=0,4$. As medidas do adolescente com assimetria de 0,3 , também em pixels, foram: $\mathrm{OD}=243,07$ e $\mathrm{OE}=224,08 ; \mathrm{E} / \mathrm{D}=0,13$ e $\mathrm{OE}=0,43$.

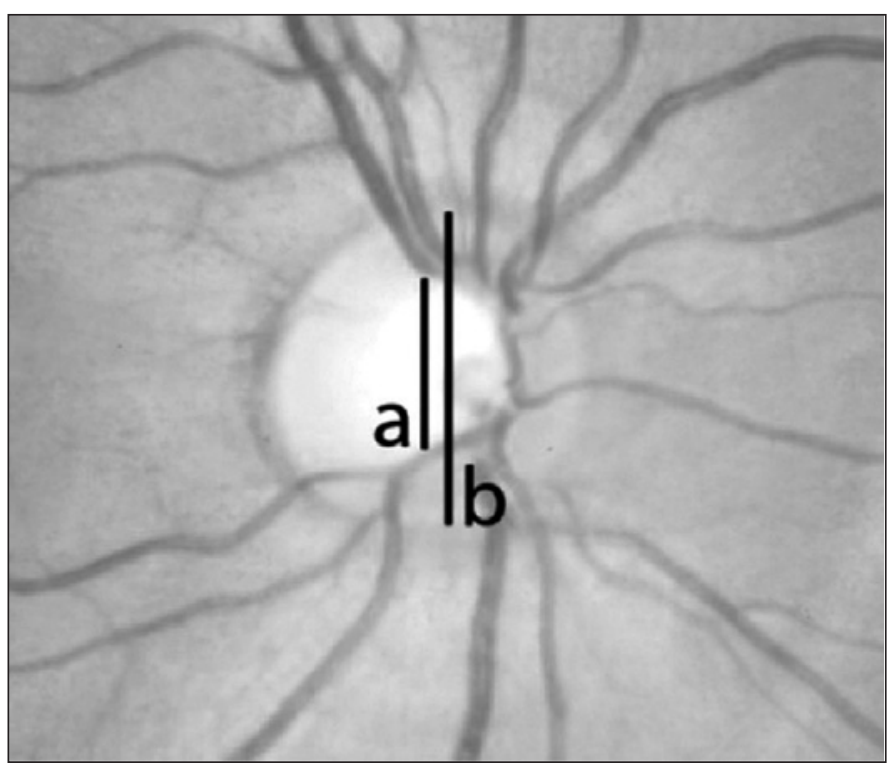

Figura 1 - Exemplo de medida do diâmetro vertical da escavação e do disco óptico de adolescente 


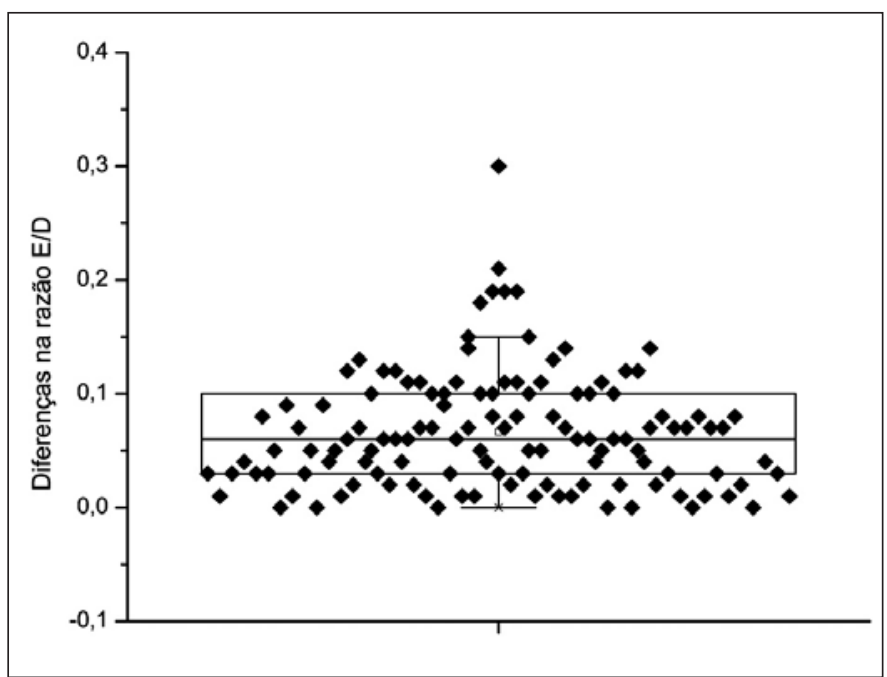

Gráfico 1 - Valores das diferenças, entre os olhos, da razão escavação disco de 123 adolescentes

As medidas refratométricas dos dois adolescentes com assimetrias na relação $\mathrm{E} / \mathrm{D}$ foram $\mathrm{OD}=-1,00$ e $\mathrm{OE}=-0,75$ dioptrias esféricas; $\mathrm{e} \mathrm{OD}=+0,75$ e $\mathrm{OE}=+0,50$ dioptrias esféricas, respectivamente.

\section{DISCUSS ÃO}

Como o estudo foi realizado em voluntários sadios, não foi conseguido o tamanho amostral pretendido $(n=250)$. Isto constituiu uma limitação do estudo. No entanto, apesar da faixa etária dos participantes, o nível de colaboração foi bom. A população inicial que aderiu ao estudo era de 125 , e somente dois não conseguiram completar os exames.

Para a verificação da relação escavação/disco podem ser utilizados diferentes métodos, como oftalmoscopia direta, biomicroscopia de fundo, estereofotografia, tomografia de coerência óptica e microscopia confocal de varredura a laser ${ }^{(5,14,19-23)}$. A avaliação das imagens fotográficas do disco óptico tem sido historicamente recomendada, tanto para o diagnóstico quanto para a detecção de progressão da doença ${ }^{(4)}$, por isso optou-se por este método, que foi adequado para os objetivos propostos. A obtenção de valores adimensionais corrigiu eventuais problemas que poderiam ter sido ocasionados por diferenças no tamanho das imagens.

Pacientes com miopia axial alta, que têm a esclera e a lâmina cribosa muito finas e, consequentemente, alterações das propriedades biomecânicas dessas túnicas oculares podem apresentar alterações na forma e no tamanho da cabeça do nervo óptico ${ }^{(24-28)}$ e maior risco de progressão do glaucoma ${ }^{(29)}$.

Em amplo estudo com base populacional, alguns autores estudando indivíduos, de diferentes etnias, verificaram que o tamanho do disco óptico pode estar diminuído quando o paciente tem vícios de refração (tanto hipermetropia quanto miopia). No entanto, essa tendência seria devida aos valores extremos dos vícios de refração, pois não há associação estatisticamente significante entre vícios de refração e área do disco, área da escavação, ou rima neural ${ }^{(14)}$.

Os diâmetros verticais dos discos ópticos dos adolescentes que apresentaram assimetrias variaram de 212,01 a 243,07 pixels (nos dois casos olhos direitos), com valores refratométricos de $-1,00$ e $+0,75$ dioptrias, respectivamente. A mediana desses diâmetros verticais, no grupo total de adolescentes estudados, foi 243,18 (Mínimo=150,05; Máximo $=619,280$ ). Considerando a proximidade da mediana e os baixos valores refratométricos não foram feitas correções dos cálculos da relação E/D.

No presente estudo, a frequência de diferenças entre as medidas da relação escavação/disco óptico, entre os dois olhos de um mesmo indivíduo não diferiu da encontrada na população adulta ocidental ${ }^{(9,15-16,22,24,29-30)}$.

Apesar disso, os dois jovens que apresentaram as maiores assimetrias, necessitam acompanhamento oftalmológico, uma vez que apresentaram um dos fatores de risco para o desenvolvimento de $\operatorname{GPAA}^{(9,31)}$.

Mesmo em pessoas sem problemas oculares, a escavação do disco óptico pode variar ao longo da vida, mas essa variação é pequena. Alguns autores realizaram estudo longitudinal, verificando aumento de 0,0026 por ano. Apesar disso, em termos individuais, o aumento pode ser significante ${ }^{(16)}$.

Por outro lado, outros autores, relatam que os parâmetros da cabeça do nervo óptico não estão relacionados com idade ${ }^{(17-18)}$.

Apesar dessa controvérsia, considera-se a importância de conhecer os valores da relação E/D em adolescentes, pois esse parâmetro, registrado em retinografias, permitirá comparações na eventualidade de os mesmos apresentarem, futuramente condições indicativas de glaucoma.

\section{CONCLUSÃO}

A frequência de assimetrias no grupo de adolescentes estudado é similar à encontrada em estudos realizados com população adulta normal.

\section{AGRADECIMENTOS}

Ao médico oftalmologista Fabrício Sleman Soubhia, pela realização das retinografias. Os autores agradecem ao Prof. Dr. Antonio Augusto Velasco e Cruz pela colaboração no delineamento do estudo e por tê-lo viabilizado, autorizando o uso de equipamentos do Laboratório de Oculoplástica da Faculdade de Medicina de Ribeirão Preto da Universidade de São Paulo. 


\section{ABSTRACT}

Purpose: To determine the frequency of asymmetries greater than 0.2 in the cup-to-disc ratio of a population of adolescents. Methods: Fundus photographs of both eyes of 123 adolescents were digitized at the resolution of 300 pixels per inch (300 dpi), 8 bits, with a RBG color pattern. The measurements were performed using the Image $\mathrm{J}$ software, version 1.42, developed by the National Institutes of Health (NIH), Java platform version 1.6. Results: The values of the differences in the cup-to-disc ratio between the two eyes of the same individual ranged from 0 to 0.30 . The mean difference was 0.07 , the median 0.06 and the standard deviation 0.05 . About $54.4 \%$ of the studied population had asymmetries up to 0.06 and $79.7 \%$ had asymmetries up to 0.1 . Two individuals presented asymmetries of more than 0.2 , corresponding to $1.63 \%$ of the evaluated subjects. Conclusion: The frequency of asymmetries in the group of adolescents studied was similar to that detected in studies of the normal adult population.

Keywords: Glaucoma/diagnosis; Optic disc/pathology; Optic nerve diseases; Fundus oculi; Visual fields; Retina; Photography/methods; Adolescents

\section{REFERÊNCIAS}

1. Foster A, Gilbert C, Johnson G. Changing patterns in global blindness:19882008. Community Eye Health. 2008;21(67):37-9.

2. Mello PA, Melo Júnior LA. Glaucoma dos nossos dias: revendo conceitos e custos. Rev Bras Oftalmol. 2003;62(9):669-77.

3. Sociedade Brasileira de Glaucoma. II Consenso Brasileiro de Glaucoma Primário de Ângulo Aberto [Internet]. Paulo Augusto de Arruda Mello, Carmo Mandía Júnior, editores. São Paulo: PlanMark; 2005.[cited 2010 Jun 1]. Disponível em: http://www.sbglaucoma.com.br/pdf/consenso02.pdf

4. Lin SC, Kuldev S, Jampel HD, Hodapp EA, Smith SD, Francis BA, Dueker DK, Fechtner RD, Samples JS, Schuman JS, Minckler DS; American Academy of Ophthalmology; Ophthalmic Technology Assessment Committee Glaucoma Panel. Optic nerve head and retinal nerve fiber layer analysis: a report by the American Academy of Ophthalmology. Ophthalmology. 2007;114(10):1937-49. Erratum in: Ophthalmology. 2008;115(3) :472. Comment in: Ophthlamology. 2008;115(7):1266-7; author reply 1267-8.

5. Sakata K, Sakata LM, Sakata VM, Santini C, Hopker LM, Bernardes R, et al Prevalence of glaucoma in a South Brazilian population: Projeto Glaucoma. Invest Ophthalmo Vis Sci. 2007;48(11):4974-9.

6. Rouland JF. [Pitfalls of examining the optic papilla (our determining a papillary excavation]. J Fr. Ophtalmol. 1999;22(1):94-6. French.

7. Sun F, Zhang X, BianX. [An ultrastructural study on human retina with late glaucoma]. Zhonghua Yan Ke Za Zhi. 1996;32(1):35-7. Chinese.

8. Chauhan BC. Comparison of strutural and functional methods II. In: Weinreb RN, Greve EL, editors. Glaucoma diagnosis. structure and function. The Hagu: Kugler publications, 2004. p.145-8.

9. Allingham RR, Shields B, Damji KF, Freedman S, Moroi SE, Shafranov G.
Shield's Textbook of Glaucoma. Philadelphia: Lippincott William \& Wilkins; 2005. p.170-96.

10. Boland MV, Quigley HA. Risk factors and open-angle glaucoma: classification and application. J Glaucoma. 2007;16(4):406-18.

11. Bron A, Chaine G, Villain M, Colin J, Nordmann JP, Renard JP, Rouland JF [Risk factors for primary open-angle glaucoma]. J Fr Ophtalmol. 2008;31(4): 435-44. French.

12. Susanna Jr. R, Medeiros FA. Nervo óptico no glaucoma. Rio de Janeiro: Cultura Médica; 2004.

13. Jonas JB, Gusek GC, Naumann GO. Optic disc, cup and neuroretinal rim size configuration and correlations in normal eyes. Invest Ophthalmol Vis Sci. 1988;29(7):1151-8. Erratum in: Invest Ophthalmol Vis Sci 1991;32(6):1893. Invest Ophthalmol Vis Sci 1992;32(2):474-5.

14. Varma R, Tielsch JM, Quigley LA, Hilton SC, Katz J, Spaeth GL, Sommer A Race-age-gender and refractive error related differences in normal optic disk. Arch Ophthalmol. 1994;112(8):1068-76.

15. Ong LS, Mitchell P, Healey PR, Cumming RG. Asymmetry in optic disc parameters: the Blue Mountains Eye Study. Invest Ophthalmol Vis Sci. 1999; 40(5):849-57.

16. Schwartz JT, Reuling FH, Garrisson RJ. Acquired cupping of the optic nerve head in normotensive eyes. Br J Ophthalmol. 1975;59(4):218-22.

17. Rizzo JF $3^{\text {rd }}$, Feke GT, Goger DG, Ogasawara H, Weiter JJ, Optic nerve head blood speed as a function of age in normal human subjects. Invest Ophthalmol Vis Sci. 1991;32(13):3263-72.

18. Pang Y, Trachimowicz R, Castell DD, Goodfellow GW, Maino DM. Optic nerve heads in pediatric African Americans using retinal tomography. Optom Vis Sci. 2009;86(12):1346-51.

19. Gherghel D, Orgui S, Prunte OS, Gugleta K, Lubeck P, Gekkieva M, Flammer J. Interocular differences in optic disc topographic parameters in normal subjects. Curr Eye Res. 2000;20(4):276-82.

20. Jonas JB, Thomas RM, George R, Berenshtein E, Muliyil J. Optic disc morphology in south India: the Vellore Eye Study. Br J Ophthalmol. 2003;87(2):189-96.

21. Min KH, Scong GJ, Hong YJ, Kim CY. Optic nerve head topographic measurements and retinal nerve fiber layer thickness in physiologic large cups. Korean J Ophthalmol. 2005;19(3):189-94.

22. Samarawickrama C, Pal A, Huynh SC, Burlutsky G, Jonas JB, Mitchell P. Measurement of optic nerve head parameters: comparison of optical coherence tomography with digital planimetry. J Glaucoma. 2009;18(8):571-5.

23. Foster PJ, Buhrmann R, Quigley HA, Johnson GJ. The definition and classification of glaucoma in prevalence surveys. Br J Ophthalmol. 2002;86(2):238- 42.

24. Daubs JG, Crick RP. Effect of refractive error on the risk of ocular hypertension and open angle glaucoma. Trans Ophthalmol Soc UK. 1981;101(1):121-6.

25. Kawase K, Tomidokoro A, Araie M, Iwase A, Yamamoto T; Tajimi Study Group. Ocular and systemic factors related to intraocular pressure in japanese adults: the Tajimi study. Br J Ophthalmol. 2008;92(9):1175-9.

26. Mitchell P, Hourihan F, Sandbach J, Wang JJ. The relationship between glaucoma and myopia: The Blue Mountains Eye Study. Ophthalmology. 1999; 106(10):2010-5

27. Oku Y, Oku H, Park M, Hayashi K, Takahashi S, Shouhi T, Chihara E. Long axial length as risk factor for normal tension glaucoma. Graefs Arch Clin Exp Ophthalmol. 2009;247(6):781-7.

28. Sociedade Brasileira de Glaucoma. III Consenso Brasileiro de Glaucoma Primário de Ângulo Aberto. Paranhos-Junior A, Omi CA, Prata-Junior JA, editores. São Paulo: BestPoint; 2009.

29. Armaly MF. The optic cup in the normal eye. i. Cup width, depth, vesse displacement, ocular tension and outflow facility. Am J Ophthalmol. 1969;68(3): 401-7.

30. Quigley HA, Brown AE, Morrison JD, Drance SM. The size and shape of the optic disc in normal human eyes. Arch Ophthalmol. 1990;108(1):51-7.

31. Leske MC, Wu SY, Hennis A, Honkaken R, Nemesure B; BESS Study Group. Risk factors for incident open-angle glaucoma: the Barbados Eye Studies. Ophthalmology. 2008;115(1):85-93. 